\title{
Teaching PCA through Letter Recognition
}

\author{
Tanja Van Hecke \\ Faculty of Applied Engineering Sciences, University College Ghent, Ghent, Belgium. \\ Email: Tanja.VanHecke@hogent.be \\ Received February $8^{\text {th }}, 2011$; revised March 14 $4^{\text {th }}, 2011$; accepted April $2^{\text {nd }}, 2011$.
}

\begin{abstract}
This article presents the use of a real life problem to reach a deeper understanding among students of the benefits of principal components analysis. Pattern recognition applied on the 26 letters of the alphabet is a recognizable topic for the students. Moreover it is still verifiable with computer algebra software. By means of well defined exercises the student can be guided in an active way through the learning process.
\end{abstract}

Keywords: Data Reduction, Eigenvalues, Eigenvectors

\section{Introduction}

Principal Components Analysis (PCA) is a statistical technique for data reduction which is taught to students mostly with a pure mathematical approach. This paper describes how teachers can introduce students to the concepts of principal components analysis by means of letter recognition. The described approach is one of an active learning environment (with hands-on exercises can be implemented in the classroom), a platform to engage students in the learning process and may increase student/student and student/instructor interaction. The activities require use of some basic matrix algebra and eigenvalue/eigenvector theory. As such they build on knowledge students have acquired in matrix algebra classes.

Former attempts to develop a more creative instruction approach for PCA can be found with Dassonville and Hahn (Dassonville, 2000). They developed a CD-rom geared to the teaching of PCA for business school students. The test of this pedagogical tool showed that this new approach, based on dynamic graphical representations, eased the introduction to the field, yet did not foster more effective appropriation of those concepts. Besides, when the program was used in self tuition mode, the students felt disconnected from the class environment, as Dassonville and Hahn claim themselves.

A second initiative is DoLStat@d (Mori, 2003), developed at Okayama University in Japan by Yuichi Mori and colleagues. This web based learning system, available online at http://mo161.soci.ous.ac.jp/@d/DoLStat/index.html, provides real world data with their analysis stories about various topics, PCA included. Since only applications are presented, without any background information about the method itself, students unfamiliar to PCA, will not reach a deeper understanding about PCA and will keep stabbing at a recipe approach.

\section{Principal Components Analysis}

The objective of PCA (Jackson, 2003) is to obtain a low-dimensional representation of the objects/individuals with minimum information loss, which facilitates compression of the initial data and extracting the most relevant characteristics.

PCA is a known data reduction technique in statistical pat- tern recognition and signal processing (Kastleman, 1996) (Turk, 1991). It is valuable because it is a simple non-parametric method of extracting relevant information from confusing datasets. PCA is also called the Karhunen-Loeve Transform (KLT, named after Kari Karhunen (Karhunen, 1947) \& Michel Loève (Loève, 1978)) or the Hotelling Transform (Hotelling, 1935).

PCA involves finding eigenvalues and corresponding eigenvectors of the data set, using the covariance matrix. The corresponding eigenvalues of the matrix give an indication of the amount of information the respective principal components represent. The methodology for calculating principal components is given by the following algorithm.

Let $x_{1}, x_{2}, \cdots, x_{m}$ be the variables.

- Computation of the global means $x_{i} \quad(i=1,2, \cdots, m)$

- Computation of the sample covariance matrix $\Sigma$ of dimension $m \times m$

- Computation of the eigenvalues and eigenvectors of $\Sigma$

- Keep only the $n$ eigenvectors $\boldsymbol{v}_{\boldsymbol{i}}=\left\{v_{i 1}, v_{i 2}, \cdots, v_{i m}\right\}$ $(i=1,2, \cdots, n)$ corresponding to the largest eigenvalues. Then $v_{i 1} x_{1}+v_{i 2} x_{2}+\cdots+v_{i m} x_{m}(i=1,2, \cdots, n)$ are called principal components.

Corresponding eigenvectors are uncorrelated and have the greater variance. In order to avoid the components that have an undue influence on the analysis, the components are usually coded with mean as zero and variance as one. This standardization of the measurement ensures that they all have equal weight in the analysis.

\section{Representation of Letters by Binary Variables}

We use the pixel representation with seven rows and five columns as in Figure 1 for the alphabet. This image is transformed into a binary vector representation (see Table 1). This was accomplished by using 35 variables $x_{i} \quad(i=1,2, \cdots, 35)$ by running the figure from top to down and from left to right assigning 1 to an occupied pixel and 0 to a non-occupied pixel.

Student exercise 1: Make the binary vector representation of the 26 letters of the alphabet (less time consuming: each student makes one).

Student exercise 2: Use Maple (www.maplesoft.com) or some 
Table 1.

The binary vector representing the letters of the alphabet.

\begin{tabular}{ccccccccccccccc}
\hline letter & $x_{1}$ & $x_{2}$ & $x_{3}$ & $x_{4}$ & $x_{5}$ & $x_{6}$ & $x_{7}$ & $x_{8}$ & $x_{9}$ & $x_{10}$ & $x_{11}$ & $x_{12}$ & $\ldots$ & $x_{35}$ \\
\hline A & 0 & 0 & 1 & 0 & 0 & 0 & 1 & 0 & 1 & 0 & 1 & 0 & $\ldots$ & 1 \\
B & 1 & 1 & 1 & 1 & 0 & 1 & 0 & 0 & 0 & 1 & 1 & 0 & $\ldots$ & 0 \\
C & 0 & 1 & 1 & 1 & 0 & 1 & 0 & 0 & 0 & 1 & 1 & 0 & $\ldots$ & 0 \\
$\ldots$ & $\ldots$ & $\ldots$ & $\ldots$ & $\ldots$ & $\ldots$ & $\ldots$ & $\ldots$ & $\ldots$ & $\ldots$ & $\ldots$ & $\ldots$ & $\ldots$ & $\ldots$ & $\ldots$ \\
Z & 1 & 1 & 1 & 1 & 1 & 0 & 0 & 0 & 0 & 1 & 0 & 0 & $\ldots$ & 1 \\
\hline
\end{tabular}
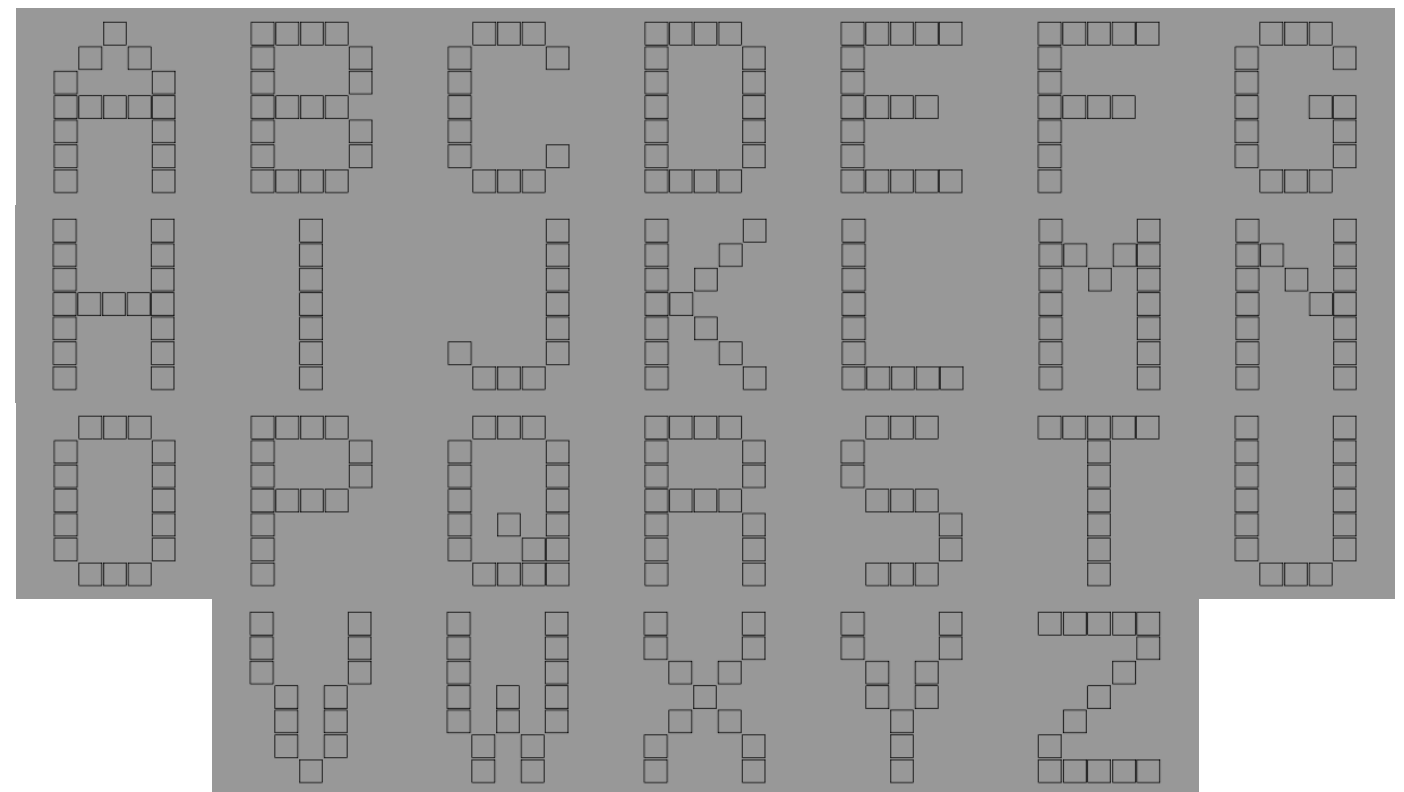

Figure 1.

The pixel representation of the 26 letters of the alphabet.

other computer algebra software to construct the covariance matrix $\Sigma$ and to obtain its eigenvalues and eigenvectors.

\section{PCA Applied on Letters}

A principal components analysis applied on this data reveals that replacing the 35 variables by the first ten principal components, explains already almost $90 \%$ of the total variance (see Figure 2). The two main principal components are displayed in Figure 3 for all letters. As PC1 and PC2 are the two most predominant eigenvalues, the distance in this two-dimensional diagram gives an indication of the resemblance of the letters. When including the first ten eigenvalues, a more general distance function can be created.

Student exercise 3: How many principal components are needed to explain $75 \%$ of the total variance?

Student exercise 4: Make a visualization of the resemblance of the letters of the alphabet defined as in Figure 1 by means of a two dimensional diagram with variables $\mathrm{PC} 1$ and $\mathrm{PC} 2$ as in Figure 3.

When receiving a letter described by the coloring of the pixel model, it is possible to detect the letter from the prescribed alphabet set that resembles most the given letter, by making

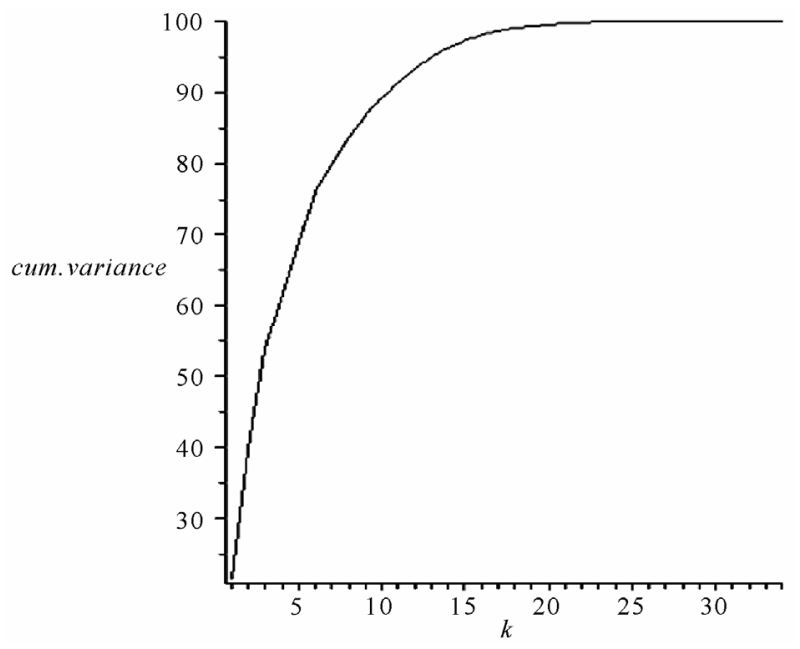

Figure 2.

The cumulative variance of the subset of the first $k$ principal components. 


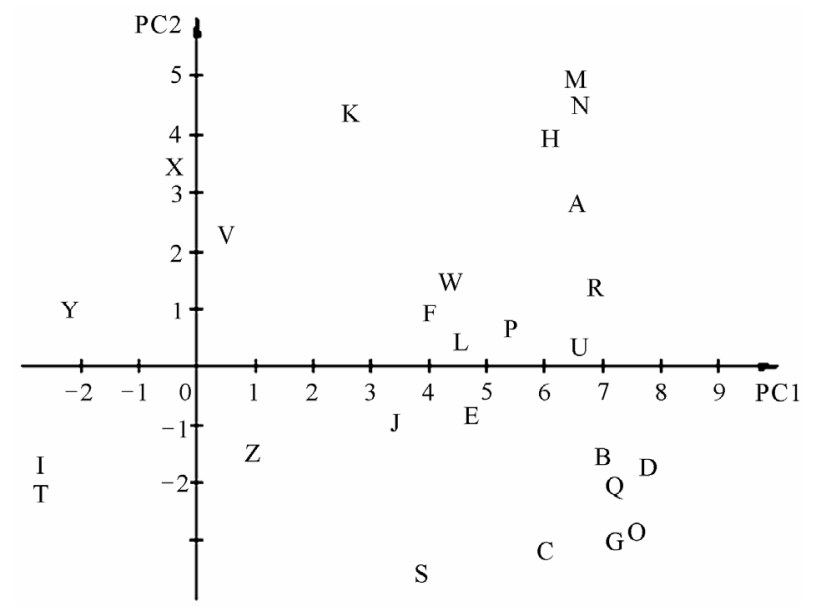

Figure 3.

The first two principal components for the 26 letters.

calculations on the distances between letters.

The distance between a first letter $l_{1}$ described by the variables $x_{i}^{1}(i=1,2, \cdots, 35)$ and a second letter $l_{2}$ described by the variables $x_{i}^{2}(i=1,2, \cdots, 35)$ is defined by

$$
d\left(l_{1}, l_{2}\right)=\sqrt{\sum_{i=1}^{35}\left(x_{i}^{1}-x_{i}^{2}\right)^{2}} .
$$

As the computational cost increases rapidly with an extensive collection of letters to choose (each represented by 35 variables), a considerable gain of effort is reached when using the principal components $p c^{1}$ and $p c^{2}$ of the letters $l_{1}$ and $l_{2}$ respectively instead. These vectors have only ten components $p c_{i}(i=1,2, \cdots, 10)$ defined by

$$
p c_{i}=v_{i 1} x_{1}+v_{i 2} x_{2}+\cdots+v_{i 35} X_{35}
$$

This means that the distance function $d$ is replaced by

$$
d_{P C}\left(l_{1}, l_{2}\right)=\sqrt{\sum_{i=1}^{10}\left(p c_{i}^{1}-p c_{i}^{2}\right)^{2}}
$$

in order to quantify the resemblance of two letters.

The letter from the prescribed alphabet with the smallest distance to the given letter $l_{1}$ can be identified with the given letter.

Example 1:

The letter $l_{1}=$ 'P' written as in Figure 4 will be recognized as the standard letter from Figure 1, as the distance $d_{P C}$ between both is only 1.21 , smaller than the distance to f.e. the standard letter R from Figure 1 which resembles $l_{1}$ as well. Their distance is 1.83 .

Example 2:

The letter $l_{1}=$ ' $\mathrm{A}$ ' written as in Figure 5 will not be recognized as the standard letter from Figure 1, as the distance between both is 3.72 , greater than the distance to the standard letter R from Figure 1 which resembles $l_{1}$ better. Their distance is 3.21 .

Example 1 supports the robustness of the described technique, where example 2 shows its limits and supports the demand for refinement. As such they are two good exercises for the students to evaluate the technique and to suggest some improvements. Students can discuss the balance that should be found between calculation cost and the quality improvement of the method by refining the pixel grid or incorporating more eigenvectors. To perform the extensive calculations the computer algebra software Maple can be used.

Student exercise 5: Investigate by means of $d_{P C}$ if standard letter S defined as in Figure 1, resembles most the letter as depicted in Figure 6.

\section{Conclusion}

My experiences with engineering students revealed the positive impulse when presenting a recognizable and interesting problem to convince students of the usefulness of mathematics and statistics. I succeeded in removing partly the prejudices conventional on mathematics. Some of the comments of the students when asking their opinion about the lesson, were:

"Apparently math is not always boring."

"Never thought there were matching points between mathe-

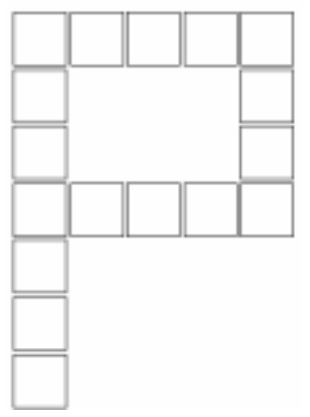

Figure 4.

Alternative representation of the letter ' $P$ '.

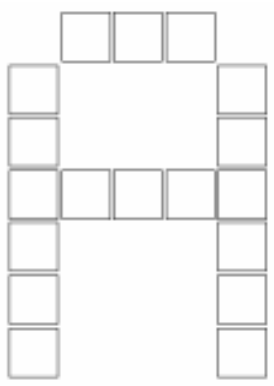

Figure 5.

Alternative representation of the letter ' $A$ '.

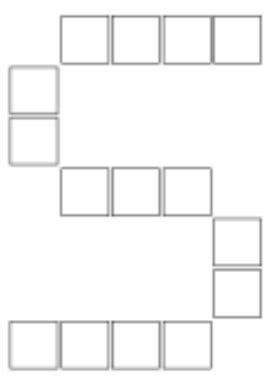

Figure 6.

Alternative representation of the letter ' $S$ '. 
matics and language."

"Surprising that we can make computers smart by means of mathematics, since they are able to recognize letters!"

"Granted, today revealed that statistics is more than calculating the mean of our exam results."

The discussions I held in class with my engineering students, resulted in an impressive improvement in their mathematical comprehension. Moreover the students became aware of the relevance of reducing the multidimensional datasets to lower dimensions for analysis by means of principal components analysis.

\section{References}

Dassonville, P., \& Hahn, C. (2000). The multimedia tool: A transitional medium beween the mathematician's culture and the professional's culture in teaching PCA in a business school. In A. Ahmed, J.
Kraemer, \& H. Williams (Eds.), Cultural diversity in mathematics Education (pp. 125-136). United Kingdom: Horwood

Hotelling, H. (1935). The most predictable criterion. Journal of Educational Psychology, 26, 139-142. doi:10.1037/h0058165

Jackson, J. E. (2003). A user's guide to principal components. New Jersey: Wiley \& Sons, Inc.

Karhunen, K. (1947). Über lineare methoden in der wahrscheinlichkeitsrechnung. Annales Academiæe Scientiarum Fennicae Series A1, Mathematica-Physica, 37, 1-79.

Kastleman, K. (1996). Digital image processing. London: Prentice Hall.

Loève, M. (1978). Probability theory Vol. II, graduate texts in mathematics 46 (4th ed.). New York, NY: Springer-Verlag.

Mori, Y., Yamamoto, Y., \& Yadohisa, H. (2003). Data-oriented learning system of statistics based on analysis scenario/story (DoLStat). Bulletin of the International Statistical Institute (ISI), 54th Session Proceedings, Volume LX Two Books, Book 2 (pp. 74-77).

Turk, M., \& Pentland, A. (1991). Eigenfaces for Recognition. Journal of Cognitive Neurosicence, 3, 71-86. doi:10.1162/jocn.1991.3.1.71 